\title{
Hypoglossal-Facial Nerve Reconstruction Using a Y-Tube-Conduit Reduces Aberrant Synkinetic Movements of the Orbicularis Oculi and Vibrissal Muscles in Rats
}

\author{
Yasemin Kaya, ${ }^{1}$ Umut Ozsoy, ${ }^{1}$ Murat Turhan, ${ }^{2}$ \\ Doychin N. Angelov, ${ }^{3}$ and Levent Sarikcioglu ${ }^{1}$ \\ ${ }^{1}$ Department of Anatomy, Akdeniz University Faculty of Medicine, 07070 Antalya, Turkey \\ ${ }^{2}$ Department of Ear Nose Throat, Akdeniz University Faculty of Medicine, 07070 Antalya, Turkey \\ ${ }^{3}$ Anatomical Institute I, University of Cologne, 50931 Cologne, Germany \\ Correspondence should be addressed to Levent Sarikcioglu; levent@akdeniz.edu.tr
}

Received 23 June 2014; Revised 16 September 2014; Accepted 17 September 2014; Published 9 December 2014

Academic Editor: Stefan Rampp

Copyright (C) 2014 Yasemin Kaya et al. This is an open access article distributed under the Creative Commons Attribution License, which permits unrestricted use, distribution, and reproduction in any medium, provided the original work is properly cited.

\begin{abstract}
The facial nerve is the most frequently damaged nerve in head and neck trauma. Patients undergoing facial nerve reconstruction often complain about disturbing abnormal synkinetic movements of the facial muscles (mass movements, synkinesis) which are thought to result from misguided collateral branching of regenerating motor axons and reinnervation of inappropriate muscles. Here, we examined whether use of an aorta Y-tube conduit during reconstructive surgery after facial nerve injury reduces synkinesis of orbicularis oris (blink reflex) and vibrissal (whisking) musculature. The abdominal aorta plus its bifurcation was harvested $(N=$ 12) for Y-tube conduits. Animal groups comprised intact animals (Group 1), those receiving hypoglossal-facial nerve end-to-end coaptation alone (HFA; Group 2), and those receiving hypoglossal-facial nerve reconstruction using a Y-tube (HFA-Y-tube, Group 3). Videotape motion analysis at 4 months showed that HFA-Y-tube group showed a reduced synkinesis of eyelid and whisker movements compared to HFA alone.
\end{abstract}

\section{Introduction}

The facial nerve is the most frequently damaged nerve due to a variety of reasons including radical parotidectomy, petrous bone surgery, removal of the cerebellopontine angle tumors, and iatrogenic injury [1-5]. The resulting facial paralysis and loss of mimetic movement is a social handicap that often leads to severe psychological and economic hardship [6].

The most common surgical approach for repairing transected nerves has been direct suture of the two stumps (endto-end anastomosis) [7] but functional recovery is poor [8]. Indeed, despite significant advances in microsurgical and neurootological techniques, functional recovery remains less than optimal $[4,8]$. Furthermore, facial palsy is complete in up to $10 \%$ of patients undergoing removal of tumors located in the cerebellopontine angle $[2,4,5,8-10]$.
In cases where large segments of the intracranial or intratemporal portions of the facial nerve are destroyed, hypoglossal-facial anastomosis (HFA) is a favoured surgical technique aimed at encouraging some innervation (hypoglossal) of the facial muscles and minimizing facial palsy $[4,11,12]$. Indeed, in animal studies, it has been shown that end-to-end anastomosis of the proximal stump of a transected intact hypoglossal nerve to the distal stump of a transected facial nerve enables outgrowth of hypoglossal axons into the paralyzed facial muscles $[10,13,14]$. However, many patients receiving HFA experience disturbing abnormal synchronous movements (synkinesis) of the facial (e.g., mimic and eyelid) muscles attributable to excessive collateral axonal branching at the lesion site $[15,16]$ and subsequent reinnervation of inappropriate (often antagonist) muscles [4, $17-20]$. 
We have previously shown that, following facial nerve injury and end-to-end anastomosis, collateral branching can be reduced by local application of neutralizing antibodies to neurotrophic factors [21] or by stabilizing microtubule synthesis with taxol [22]. However, neither treatment improved recovery of facial motor (whisking) function [23]. Similarly, surgical reconstruction using a Y-tube conduit to direct regeneration of the proximal stump of the hypoglossal nerve into the distal stump of the facial nerve and avoid tightly suturing (anastomosis) the nerve stumps [24] reduced axonal sprouting but also did not improve recovery of whisking [25].

In human patients receiving facial nerve reconstruction surgery, the most common synkinesis is eyelid closure during a smile. However, little is known about the effects of different types of surgical reconstruction on the likelihood of synkinesis after nerve injury.

Rats provide an ideal model to examine synkinesis because they display spontaneous bursts of activity of the vibrissal muscles (whisking) that can be analyzed in relation to eyelid closure (blink) $[9,23,26,27]$. In intact rats, explorative whisking is not accompanied by synkinetic eyelid closure. Likewise, an "air puff" to the cornea and periorbital region triggers an eyelid closure (blink reflex) but no associated whisking of the vibrissal hairs. During facial nerve regeneration, however, excessive collateral branching of axons causes misrouting leading to aberrant reinnervation of musculature and pathological synchronized movement (synkinesis) of the orbicularis oculi [9] and vibrissal muscles [23].

We therefore examined the extent of synkinesis after facial nerve reconstruction using HFA alone or HFA using an aorta Y-tube conduit. We undertook two quantitative motion analyses: one on eyelid closure during spontaneous whisking and the other on vibrissal whisking during a blink reflex elicited by an air puff to the cornea and periorbital region. Video recordings taken at the time of data collection for a previously published experiment [25] were used for analysis.

\section{Material and Methods}

2.1. Animals. The data were collected, but not previously reported, from animals used in an earlier study [25]. Fortyeight young adult female inbred rats (origin Harlan Laboratories Israel, strain "Wistar," F11 generation) were obtained from the Laboratory Animal Unit of the Akdeniz University. Twelve animals were used for harvesting their abdominal aorta plus its bifurcation. The remainder were split into 3 groups $(n=12$ in each) comprising intact controls (Group 1), rats subjected to hypoglossal-facial nerve end-toend coaptation alone (HFA; Group 2), and those receiving hypoglossal-facial nerve reconstruction using a Y-tube (HFAY-tube, Group 3). After 4 months, all animals were subjected to video-based motion analysis of orbicularis oculi and vibrissal motor performance.

Throughout, allocation was concealed; that is, the person undertaking the surgery did not know to which group the animal would be allocated. Animals were randomised into groups using a randomised number sequence and assessment was blinded.

\subsection{Surgical Procedures}

2.2.1. Preparation of Y-Tube. The aortal bifurcation into the right and left common iliac arteries was used as a Y-tube conduit. In brief, once exposed, an artery clamp was applied to the aorta just below the diaphragm to avoid excessive bleeding. The aorta was cut and traced inferiorly taking care to avoid punctures and a $1.5-2.0 \mathrm{~cm}$ long segment including the common iliac arteries removed and irrigated with phosphate buffer to remove blood clots. Small branches originating from the aorta were cauterized.

2.2.2. Nerve Reconstruction. Animals were anesthetized with a mixture of ketamine $(100 \mathrm{mg} / \mathrm{kg}$, i.p.) and xylazine $\mathrm{HCl}$ (15 mg/kg; i.p.). Hypoglossal-facial anastomosis (HFA; Group 2) was performed unilaterally (right side) as described previously [10]. In brief, after exposure under an operating microscope with fiberoptic illumination (Olympus SZ61), the glandula lacrimalis extraorbitalis was retracted laterally to approach the proximal part of the buccal and zygomatic branches. The facial vein crossing over the zygomatic and buccal branches was cauterized and the zygomatic and buccal branches were cut at the level of their origin from main trunk of the facial nerve. The remaining part of the facial nerve trunk was ligated with 9.0 silk suture. The hypoglossal nerve was approached by retraction of the posterior belly of the digastric and stylohyoid muscles. The hypoglossal nerve was cut just before its division into medial and lateral branches. The proximal end of the hypoglossal nerve was directly apposed (i.e., no interstump distance) to the distal stump of the facial nerve and sutured with two 9.0 sutures.

HFA using a Y-tube has also been described previously $[10,25]$. Procedures were as above except that, to avoid any delay to implantation, Y-tube preparation from one set of animals and exposure of nerve branches from the other set were accomplished simultaneously by 2 separate surgical teams. In addition, alignment of the proximal hypoglossal nerve stump and the distal facial nerve stump within the Ytube involved an interstump distance of $0.5 \mathrm{~cm}$. The Y-tube was shortened to match the interstump distance of $0.5 \mathrm{~cm}$. The proximal stump of hypoglossal nerve was entubulated into the long branch of the aorta-Y-tube conduit. The distal stumps of the zygomatic and buccal nerve branches were then entubulated into the short branches of the aorta-Y-tube conduit. Two 10.0 sutures were used to secure each stump to the Y-tube (Figure 1).

2.3. Natural Whisker Movements. Rodents make a number of different movements with their whiskers, namely, (i) large-amplitude "explorative" sweeps of the vibrissae (in the frequency range of 5-11 Hz); (ii) low-amplitude "foveal" or "palpating" whisker movements at 15-25 Hz [28, 29]; and (iii) denervation-induced tremor that occurs after facial nerve transection [30]. All whisker movements are elicited by contractions of the intrinsic and extrinsic vibrissal musculature 
Hypoglossal-facial nerve end-to-end coaptation (HFA, Group 2)

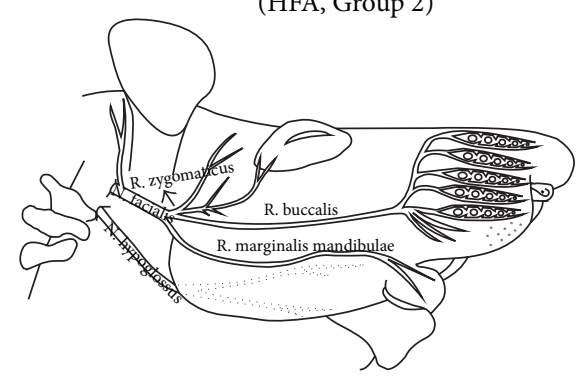

Hypoglossal-facial nerve reconstruction using a Y-tube

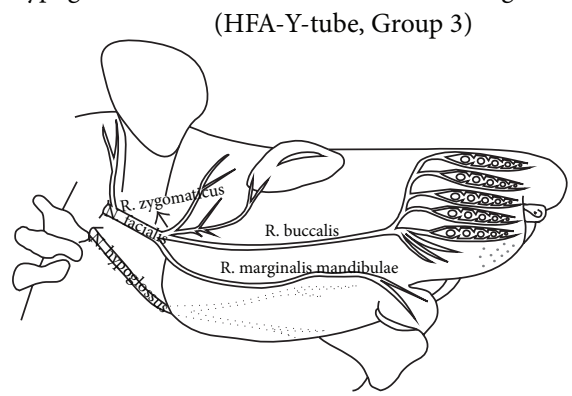

(a)
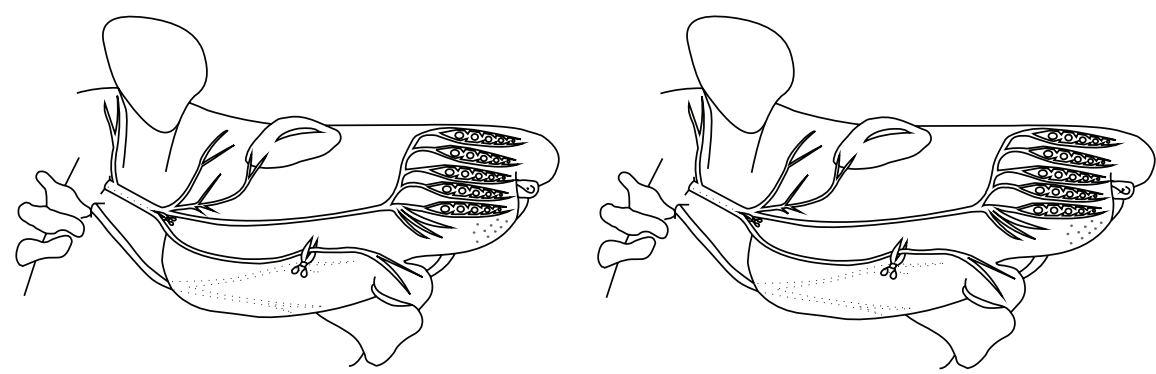

(b)
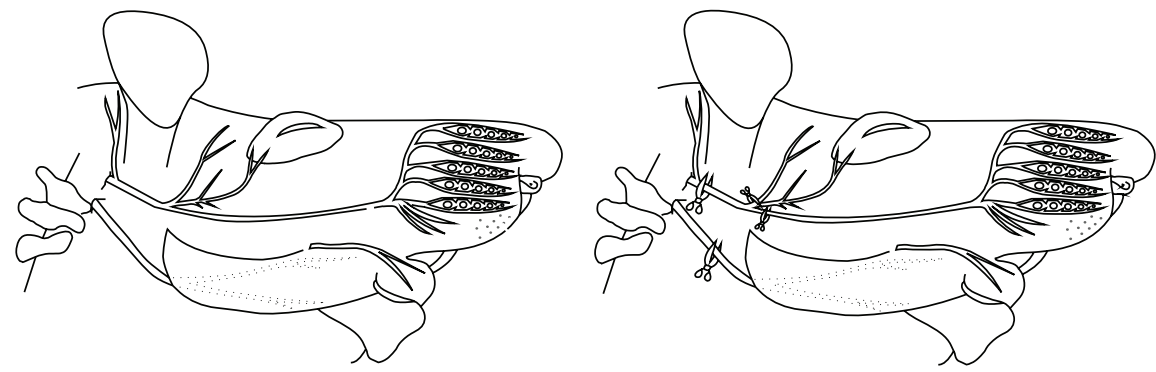

(c)
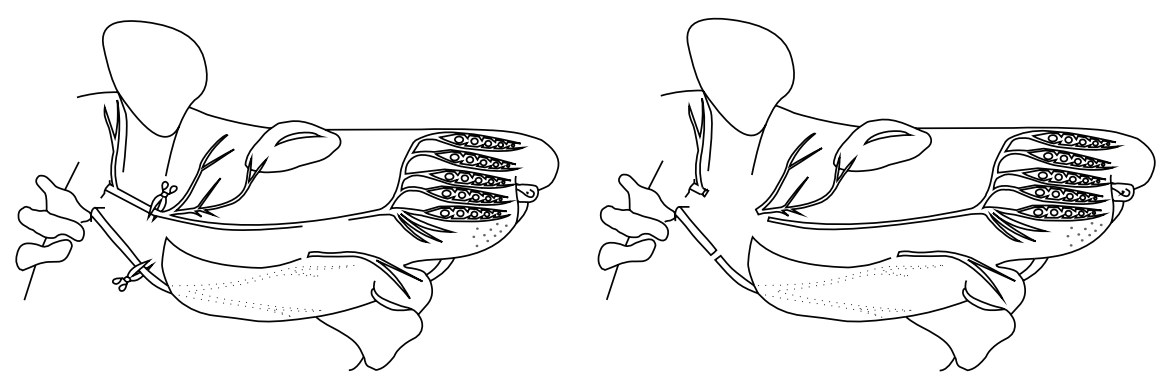

(d)
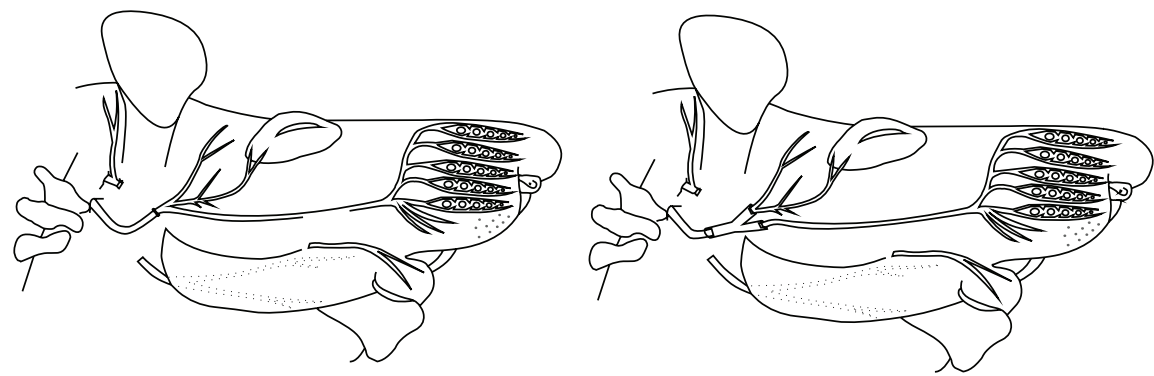

(e)

Figure 1: Drawing of the surgical procedures in Groups 2 and 3. (a) Normal anatomy of the facial and hypoglossal nerves, (b)-(d) steps of the nerve reconstruction. (e) Final step for hypoglossal-facial nerve reconstruction by using Aorta-Y-tube conduit or classical coaptation. 
which are controlled solely by the facial nerve [31]. In this study, we analyzed only the large amplitude exploratory sweeps. Following facial nerve transection, such exploratory movements are completely abolished. However, with time, and as we show here, there is a gradual progression to varying levels of motor performance which can be readily assessed by video-based motion analysis. The technique is an entirely noninvasive approach to monitor recovery of function after facial nerve repair and avoids the use of invasive electromyography.

\subsection{Simultaneous Monitoring of Eyelid and Whisker Move-} ments. Video-based motion analysis of vibrissal motor performance and eyelid closure has been previously established and examined in a series of recent experiments $[9,10,32$, 33]. However, these studies did not examine the degree of synkinesis between whisker and eyelid movements.

Four months after nerve reconstruction, all animals were videotaped for 3-5 min during active whisker exploration using a digital video camera (Sony Handycam DCR-SR70 HDD Camcorder). Care was taken to handle the animals gently to avoid stress on the animal. For calibration, a ruler laid $20 \mathrm{~cm}$ below the recorder (i.e., at a constant angle of $180^{\circ}$ ) was videotaped before each trial. Selected sequences containing the most prominent whisks (vibrissal bouts) on the intact side contralateral to surgery were captured by a 2D/Manual Advanced Video System (WinAnalyze, Mikromak Service, Berlin, Germany). Frame rate of the video analysis was 25 frame/second. Eyelid closures were assessed from the same videotape sequences.

To assess whisker movements, we used a geometrical model consisting of three reference points: (i) a point in the medial sagittal line close to the end of the nose, (ii) a point corresponding to the medial angle of the left orbita, and (iii) a point corresponding to the medial angle of the right orbita. Using this model we collected and evaluated data on the (i) whisking frequency (cycles of protraction and retraction per second), (ii) angle at maximal protraction (the rostrally open angle between the midsagittal plane and the hair shaft in degrees), and (iii) amplitude (the difference between maximal retraction and maximal protraction in degrees) (Figures 2(a) and 2(b)). The maximal retraction angle (in degrees) was subtracted from the maximal protraction angle and divided by the mean whisking amplitude obtained from intact animals. Values from intact animals were used as the denominator because animals undergoing facial nerve reconstruction have impaired function. This value was used to estimate whisker movements in comparison to intact animals. Normal whisking amplitude was considered to be $100 \%$.

To assess spontaneous eyelid movements, video sequences were inspected on screen. In addition to the reference points used for evaluating whisking, we also added a reference point half-way along the rim of each eyelid to measure the mean distance between both eyelids. Frames were selected when eyelid closure on the intact left side was maximal, that is, when the distance between the eyelids was smallest (Figure 3). The minimal distance between the upper and lower eyelids ("inter-eyelid distance") during a blink was subtracted from the intereyelid distance at rest and divided by the intereyelid distance at rest (formula in (1)). A full blink during whisking was considered to be $100 \%$ synkinesis of the orbicularis oculi muscle (Figure 4). Since eyelid closure in intact animals is complete (100\%), any increase in the intereyelid distance indicates functional impairment.

The following are formulas used to calculate synkinetic movements:

ratio of synchronous movement of the eyelid during whisking

$$
\begin{aligned}
= & \frac{\text { intereyelid distance }(\text { in } \mathrm{mm} \text { ) in rest position }- \text { minimal intereyelid distance (in } \mathrm{mm} \text { ) while maximal protraction of vibrissal hairs }}{\text { intereyelid distance (in } \mathrm{mm} \text { ) in rest position }} \\
& \times 100 \text {, }
\end{aligned}
$$

ratio of synchronous movement of the vibrissal hair during blink reflex

$\underline{\text { maximal retraction angle (in degree) in rest position - maximal protraction angle (in degree) while minimal intereyelid distance }}$ mean whisking amplitude (in degree) in intact animals

$\times 100$.

To assess elicited eyelid movements, we used a customdesigned apparatus that delivered a single standardized volume of $20 \mathrm{~mL}$ air as a "puff" to the cornea and periorbital region bilaterally at a distance of $3 \mathrm{~cm}$. Ten puffs were delivered sequentially over $120 \mathrm{~s}$. Eyelid closure was evaluated as above.

All frames of our video material were evaluated to find the highest amplitude of the vibrissal hairs during blink reflex (contraction of the orbicularis oculi muscle) or vice versa. This is why we just concentrate to find spatial relation between whisker movement and eyelid contraction on the same sequence of the movements (the full length of the movement could be observed in 3-10 consecutive frames). Full length of the video material was examined and intereyelid distance and amplitude (in degree) of the vibrissal hair movement were measured in all sequences of the movements. 


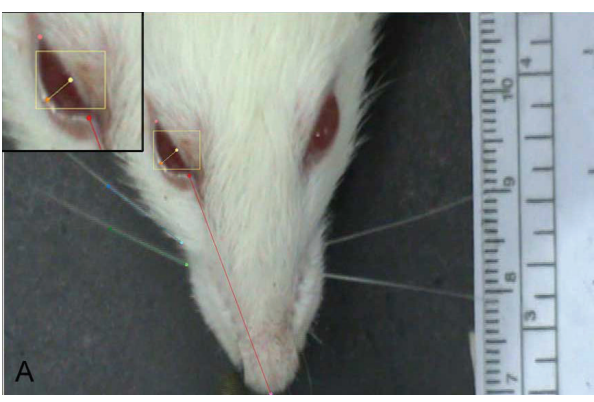

(a)

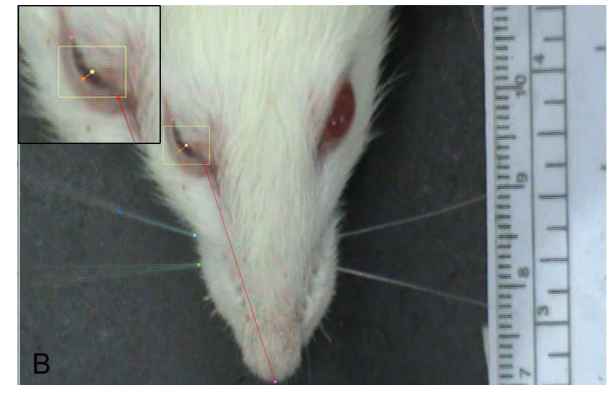

(b)

Figure 2: Consecutive frames of a video material showing synkinetic movement of the eyelid during whisking. Nerve reconstruction was performed on the right side of the animal. (a) Position of the vibrissal hairs and eyelid at rest. (b) Cocontraction of the orbicularis oculi muscle during exploratory whisking (protraction) of the vibrissal hairs.

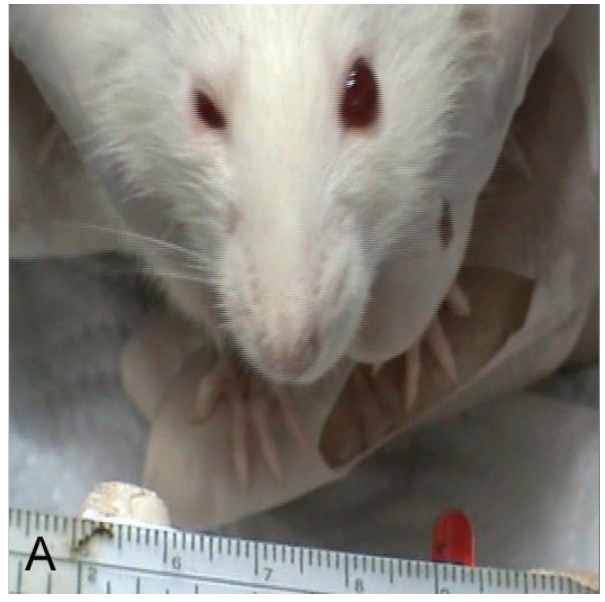

(a)

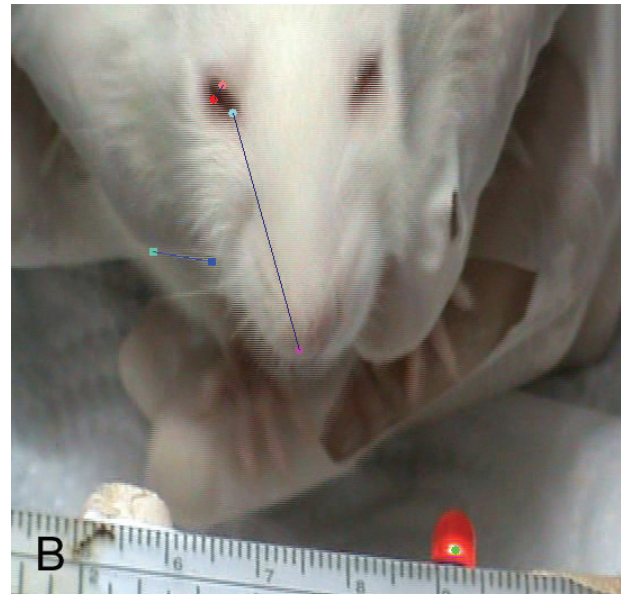

(b)

Figure 3: Consecutive frames of a video material showing eye closure stimulated by a "puff" stimulation and whisking at the same time. Nerve reconstruction was performed on the right side of the animal. (a) Position of the eyelids before starting the air puff. (b) Cocontraction of the orbicularis oculi muscle and vibrissal muscle during air puff stimulation.

For instance, when an animal contracted its orbicularis oculi muscle and showed a minimal intereyelid distance, we also measured the angular changes of the vibrissal hair to find the amplitude (we measured the retraction angle of the vibrissal hair before starting the movement and highest protraction angle while minimal intereyelid distance was observed).

It should be noted that there was a negative correlation in calculation paradigms of synkinetic movements. Minimal intereyelid distance (in $\mathrm{mm}$ ) means more synkinetic movement. In other words during whisking if the animals show full close of its eyelid, we consider that the animal showed $100 \%$ synkinesis. However maximal protraction angle value (i.e., whisking amplitude) means more synkinetic movement.

Movements of eyelid and vibrissal hairs are reverse in direction. Function of the eyelid is to diminish palpebral rima, but function of the vibrissal muscles is to move the hairs in forward direction, for example, enlarge the amplitude. In intact animals smaller intereyelid distance will mean good function (this means that orbicularis oculi muscle contracted to decrease intereyelid distance), but larger amplitude of the vibrissal hairs will mean good function (this means that vibrissal muscle contracted and moved the vibrissal hairs in forward direction). In other words if some axons in the buccal branch showed collateral axonal branching and reinnervated both of the whisker pad and orbicularis oculi muscles, smaller intereyelid distance measured during whisking will mean more synkinesis (Figure 4).

Data Analysis. Data were presented as mean \pm standard deviation and analyzed by ANOVA (post hoc Tukey test). The level of significance was set to $P<0.05$. For analysis, GraphPad Prism version 5.0 (GraphPad Software, Inc, San Diego, Calf) was used.

\section{Results}

3.1. Less Synkinetic Movements of the Eyelid during Whisking in the HFA-Y-Tube Group. The formulas which were used to calculate synkinetic movements of the eyelid and vibrissal muscles are shown in (1). 


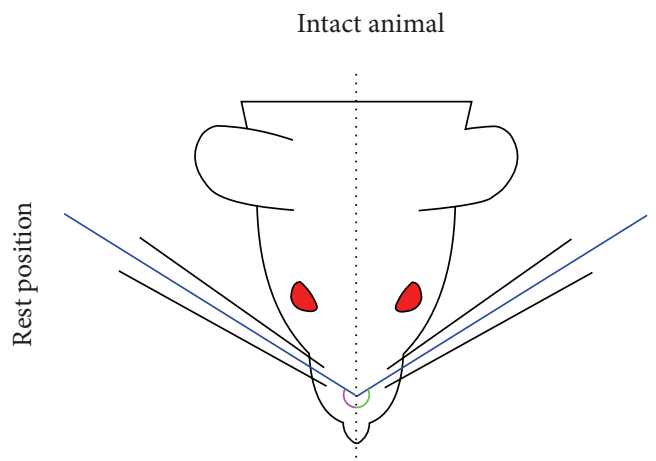

Midsagittal plane

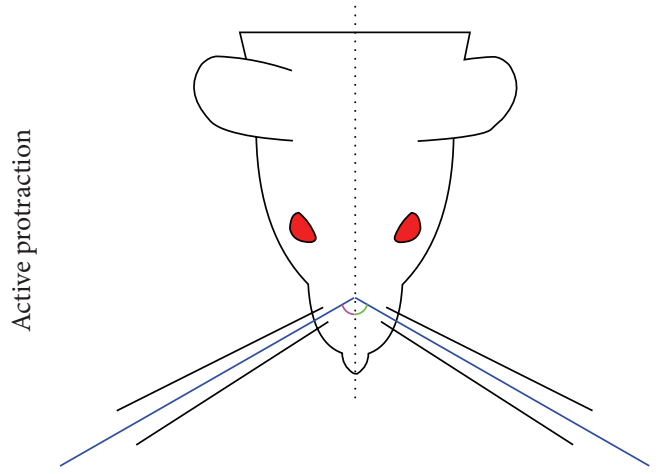

Animal with synkinetic movement

of the orbicularis oculi muscle

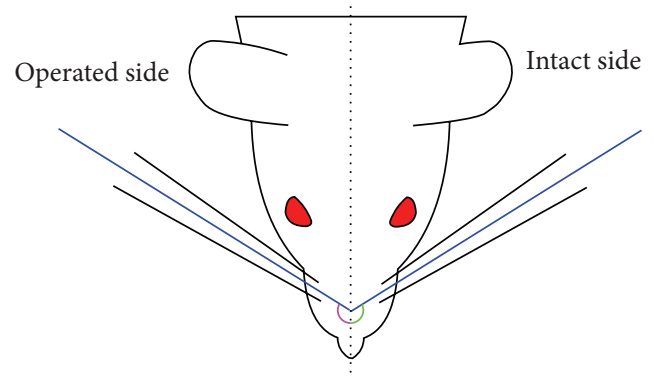

Midsagittal plane

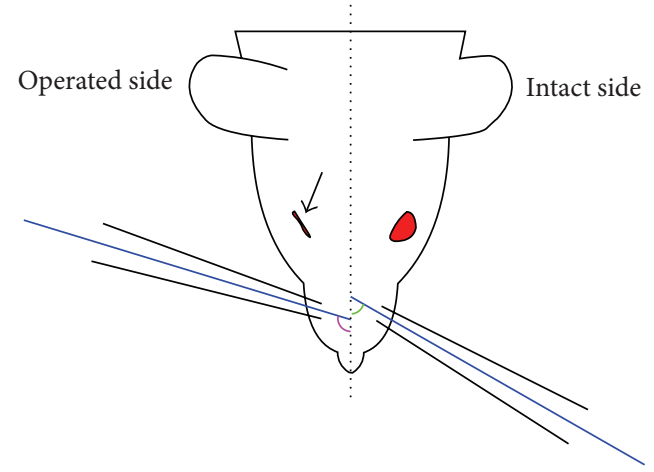

FIGURE 4: Schematic drawing of an animal showing 100\% synkinetic movement of the eyelid during whisking. Arrow shows a full blink (minimal intereyelid distance) during protraction of the vibrissal hairs.

Synkinetic movements of the eyelid during whisking were analyzed in the frame of first experimental set. The ratio (\%) of synkinetic eyelid closure occurred at the same time as whisking movement was significantly lower in the HFA+Ytube group $(27.8 \pm 3.6 \%$, mean $\pm \mathrm{SD})$ compared to the HFA group $(39.6 \pm 5.8 \%, P<0.05$, Figure 5$)$.

3.2. Less Synkinetic Movement of the Vibrissal Hairs during Eye-Closure in the HFA-Y-Tube Group. Synkinetic movements of the vibrissal muscles during blink reflex were analyzed in the frame of second experimental set.

Synkinetic movement ratios were $42.4 \pm 6.5 \%$ and $29.2 \pm$ $3.7 \%$ in HFA-coaptation and HFA-Y-tube groups, respectively. There was a significant difference between both groups (Figure 5). Synkinetic movement ratios were significantly lower $(P<0.05)$ in the HFA-Y-tube conduit group compared to those of HFA-coaptation group.

\section{Discussion}

In this study, we examined the function of vibrissal and orbicularis oculi muscles after two types of hypoglossal-facial reconstruction using an objective and quantitative analysis of simultaneous movements of both muscle groups, that is, synkinesis. We showed that synkinesis was reduced following hypoglossal-facial nerve reconstruction (HFA) using a Ytube compared to HFA-coaptation, that is, direct anastomosis, alone. Evaluation of synkinesis of eyelid and vibrissal movements may be a useful model with clinical relevance for assessing functional recovery after facial nerve injury.

4.1. Technical Issues. Direct measurement of orbicularis oculi electromyography in conjunction with search coil tracking in a magnetic field has been used previously as an indicator of eyelid motor activation and lid movements, respectively [34-36]. However, these techniques are invasive and have the disadvantage of potential morbidity from hardware implantation and chronic maintenance in soft tissues as well as challenges in relating EMG signals to palpebral fissure (upper and lower eyelid position). Our choice was therefore to analyze muscle function by noninvasive quantitative and objective video-based motion analysis, a well-established technique in our hands, for example, $[10,25,27]$, as an indirect measure of orbicularis oculi and vibrissal muscle function.

A critical feature of our video-based assessment is to measure the amplitude of natural vibrissal bouts during free exploration. Earlier studies have shown that, during free exploration, rats rhythmically move their heads so as to bring microvibrissae into contact with the surfaces they are exploring. These periodic "microvibrissal placements" are 


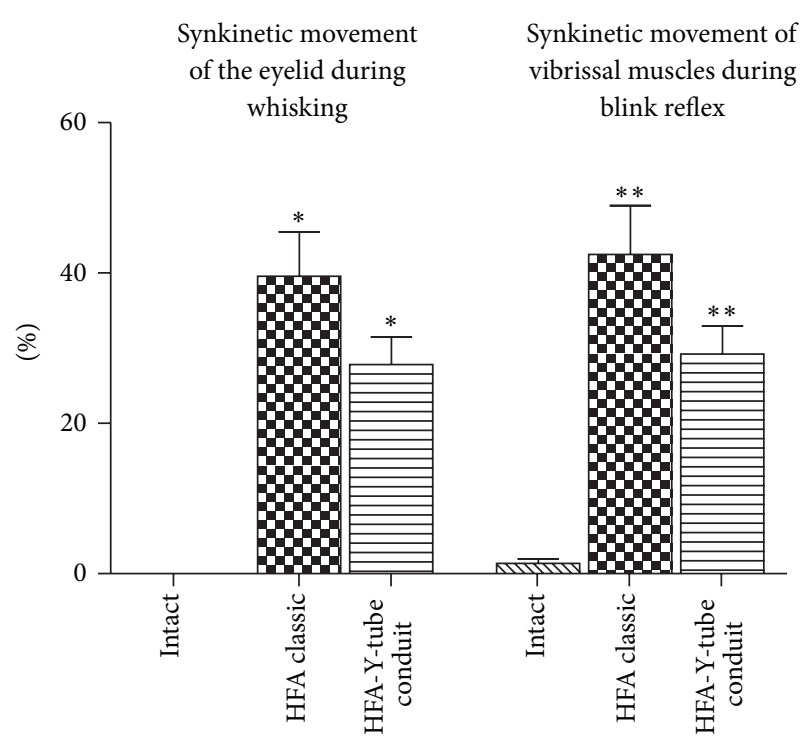

FIGURE 5: Synkinetic movement of the eyelid during whisking and synkinetic movement of the vibrissal muscles during blink reflex in two hypoglossal-facial nerve reconstruction techniques.

synchronized with macrovibrissal movements not only during free exploration, but also during texture discrimination tasks [37, 38]. As a result, during our 3-5-minute video sessions, natural head movements result in some variability in whisking effort [39].

However, if the head is immobilized, the finely-tuned physiological synchrony of micro- and macrovibrissal whisking is impaired thereby introducing a confounder. Thus, in an attempt to compensate for the resulting decrease in sensory stimuli, rats react with significantly larger than normal, and therefore nonphysiological, vibrissal excursions of the order of 90 degrees [40-43].

4.2. Possible Role of the Retractor Bulbi Muscle on Blink Reflex. Immediately after facial nerve lesion, the retractor bulbi extraocular musculature has been shown to support blink response in rabbits [44] as well as in cats [35, 45] with its increasing activity being thought to compensate for lost orbicularis oculi function. Extraocular muscles are innervated by axons originating in the accessory abducens nucleus [45-47], not the facial nerve, and are thus spared during facial nerve lesion. Similar retractor bulbi muscles have been described in rats $[47,48]$, although their role in supporting blinking behavior has not been described. Further experiments will be required to assess the possible role of the retractor bulbi muscles in our model.

4.3. Evaluation of Synkinesis after Peripheral Nerve Injury. Synkinesis following nerve injury refers to involuntary contraction of some muscles during voluntary movement of others, for example, involuntary eye closure at the same time as smiling. This phenomenon is thought to arise from axonal misrouting during regeneration of the facial nerve
[49]. Treatment options include physical therapy [50, 51], botulinum toxin injections [52], and surgical reconstruction $[53,54]$. However, these have limited success and resulting peripheral facial paralysis has profound psychologic and social impacts on patients [55-57].

Improving clinical outcome requires reliable and reproducible experimental models of peripheral nerve injuries and their reconstruction $[55,58,59]$. In addition, appropriate outcome measures are also important. Various morphologic, electrophysiologic, biochemical, and functional methods have been used to assess peripheral nerve regeneration $[60,61]$. However, results are variable making interpretation difficult $[55,57,58,61]$. Although functional methods appear to relate to the success of neural regeneration, it has been difficult to establish objective and reproducible functional measures [60].

Another important issue is that we could not understand whether recovery from facial nerve cut causes abnormal whisker movements desynchronization of whisker movement, more side to side asymmetry. Although we tried to measure abnormal coactivation (contraction) of muscles, it is possible that there may be abnormal whisker synchronization. Cortical motor neurons can drive whisker movement but only of a few whiskers $[62,63]$, and facial motor neurons have various connections to muscles $[62,64]$, so it is likely the reinnervation would produce abnormal synchrony of whisker movement, not just synchronous contractions that do not normally occur. Therefore we think the further studies should be done to understand this important issue of the nerve recovery.

4.3.1. Grading Systems for Evaluation of Synchronous Movements. Facial nerve palsy can be scored clinically using the Sunnybrook, House-Brackmann, and Yanagihara grading systems whose reliability has also been established [65]. Scoring systems have also been developed for animal models. Synchronous movements ( $0=$ none; $3=$ normal $)$ of the upper lip and eyelids have been assessed in rabbits after facial nerve injury and encompass spontaneous movements as well as those elicited by light touch of the forehead and nose [66]. Another scoring system has also been developed in rats following facial nerve injury $(0=$ no movement; $100 \%=$ full movement) [67]. However, both systems involve stimulating the eyes and vibrissae together and are subjective.

A different scoring system [68] has been developed to assess vibrissal position and eyelid closure separately but still involves a subjective ordinal scale (absence of eyelid closure $=$ 1 ; presence of orbicular muscle contraction, without blinking reflex $=2 ; 50 \%$ eyelid closure through blinking reflex $=3$; $75 \%=4 ; 100 \%$ eyelid closure and blinking reflex $=5$. Absence of movement and posterior position of the vibrissae $=1$; slight shivering and posterior position $=2$; greater shivering and posterior position $=3$; normal movement with posterior position $=4$; symmetrical movement of the vibrissae, with anterior position $=5$ ).

In a recent study, Hadlock et al. [69] described a method to establish normative parameters for both spontaneous and 
induced whisking and blinking behavior after facial nerve manipulation. Before recording the whisking and blinking behavior, they used a titanium head fixation device secured to the animal's cranium with titanium screws, with each of its four posts emerging through a separate stab incision in the skin. So, the animals were in a very stressful situation (in head-fixed position). From those animals, they continuously recorded right and left $\mathrm{C}-1$ whisker positions of the animals for 5-minute sessions and continuously recorded changes in infrared detection corresponding to eye closure. They elicited whisking and blinking by delivery of olfactory stimuli (10 s scented airflows) and corneal air puffs. They reported that air puff elicited an ipsilateral blink 99\% of the time, a contralateral blink $18 \%$ of the time, and changes in, or initiation of, bilateral whisking $70 \%$ of the time. Olfactory stimulus delivery prompted a change in whisking behavior $83 \%$ of the time and eye closure $20 \%$ of the time. In agreement with this study, we consider that the frequency (how often) of vibrissal and eyelid movements is important. However, in the present study we focused on measuring the synkinesis of eye closure and whisking in different experimental sets. We think that the intensity of the synkinetic movement is more important factor. Analysis of synkinetic movements of the two main muscle groups of the face may provide valuable data on understanding recovery movements after facial nerve injury. Additionally we think that there is no need to setup such sophisticated systems, that is, laser system, to measure the synkinesis. We also proved that our video-based motion analysis system can be used to analyze synkinetic movements.

\section{Conclusions}

In our previous study [25], we found that HFA+Y-tube reduced collateral axonal branching. This reduction, however, did not result in improved whisking function (increased amplitude) nor in reduced polyinnervation of the levator labii superioris muscle [25]. We now extend these studies and show that reduced synkinesis of the orbicularis oculi and vibrissal muscles after HFA+Y-tube conduit correlates with the reduction in collateral axonal sprouting shown previously in the same animals [25].

In conclusion, the analysis of our video material revealed that reconstruction of the hypoglossal nerve with an injured facial nerve using a Y-tube conduit results in lower synkinetic activities of the orbicularis oculi and vibrissal muscles compared to when reconstruction is undertaken using simple end-to-end anastomosis. Surgical reconstruction that enables transected axons to regenerate in an unforced manner towards their targets via a Y-tube conduit may both reduce collateral axonal branching and synkinetic movement and improve function.

\section{Conflict of Interests}

The authors declare that there is no conflict of interests regarding the publication of this paper.

\section{Authors' Contribution}

Doychin N. Angelov and Levent Sarikcioglu equally contributed to this work and shared last authorship.

\section{Acknowledgments}

This study was supported by Akdeniz University Research Fund (Project number 2013.01.0103.010). The authors also thank Mr. Erol Nizamoglu, Mr. Ibrahim Caliskan, and Vet. Doga Besne for their technical assistance.

\section{References}

[1] D. A. Bascom, B. M. Schaitkin, M. May, and S. Klein, "Facial nerve repair: a retrospective review," Facial Plastic Surgery, vol. 16, no. 4, pp. 309-313, 2001.

[2] A. Gharabaghi, S. Heckl, J. Kaminsky et al., "Hirnnervenausfälle durch ungewöhnliche Schädelbasisläsionen des Sinus cavernosus," HNO, vol. 55, no. 4, pp. 278-280, 2007.

[3] O. Guntinas-Lichius, "The facial nerve in the presence of a head and neck neoplasm: assessment and outcome after surgical management," Current Opinion in Otolaryngology and Head and Neck Surgery, vol. 12, no. 2, pp. 133-141, 2004.

[4] G. Hundeshagen, Effect of Mechanical Stimulation of the Vibriseal Muscles on the Recovery of Whisking after HypoglossalFacial Nerve Anastomosis (HFA) in Adult Rats, Department of Anatomy Cologne: University of Cologne, 2006.

[5] S. Sood, R. Anthony, J. J. Homer, P. Van Hille, and J. D. Fenwick, "Hypoglossal-facial nerve anastomosis: assessment of clinical results and patient benefit for facial nerve palsy following acoustic neuroma excision," Clinical Otolaryngology and Allied Sciences, vol. 25, no. 3, pp. 219-226, 2000.

[6] A. Miehlke, "Reconstruction of function in vocal cord paralysis. Round table discussion," Laryngologie, Rhinologie, Otologie, vol. 65, no. 1, pp. 1-4, 1986.

[7] N. J. Naff and J. M. Ecklund, "History of peripheral nerve surgery techniques," Neurosurgery Clinics of North America, vol. 12, no. 1, pp. 197-209, 2001.

[8] J. D. F. Kerrebijn and J. L. Freeman, "Facial nerve reconstruction: outcome and failures," Journal of Otolaryngology, vol. 27, no. 4, pp. 183-186, 1998.

[9] A. Bischoff, M. Grosheva, A. Irintchev et al., "Manual stimulation of the orbicularis oculi muscle improves eyelid closure after facial nerve injury in adult rats," Muscle \& Nerve, vol. 39, no. 2, pp. 197-205, 2009.

[10] O. Guntinas-Lichius, G. Hundeshagen, T. Paling et al., "Manual stimulation of facial muscles improves functional recovery after hypoglossal-facial anastomosis and interpositional nerve grafting of the facial nerve in adult rats," Neurobiology of Disease, vol. 28, no. 1, pp. 101-112, 2007.

[11] K. Hwang, S. G. Kim, and D. J. Kim, "Facial-hypoglossal nerve anastomosis using laser nerve welding," Journal of Craniofacial Surgery, vol. 17, no. 4, pp. 687-691, 2006.

[12] E. Stennert, "I. Hypoglossal facial anastomosis: Its significance for modern facial surgery. II. Combined approach in extratemporal facial nerve reconstruction," Clinics in Plastic Surgery, vol. 6, no. 3, pp. 471-486, 1979.

[13] D. N. Angelov, A. Gunkel, E. Stennert, and W. F. Neiss, "Recovery of original nerve supply after hypoglossal-facial anastomosis causes permanent motor hyperinnervation of the whisker-pad 
muscles in the rat," Journal of Comparative Neurology, vol. 338, no. 2, pp. 214-224, 1993.

[14] Y.-S. Chen, C.-J. Hsu, T.-C. Liu, N. Yanagihara, and S. Murakami, "Histological rearrangement in the facial nerve and central nuclei following immediate and delayed hypoglossalfacial nerve anastomosis," Acta Oto-Laryngologica, vol. 120, no. 4, pp. 551-556, 2000.

[15] J. H. Morris, A. R. Hudson, and G. Weddell, "A study of degeneration and regeneration in the divided rat sciatic nerve based on electron microscopy - II. The development of the "regenerating unit'”' Zeitschrift für Zellforschung und Mikroskopische Anatomie, vol. 124, no. 2, pp. 103-130, 1972.

[16] G. D. Shawe, "On the number of branches formed by regenerating nerve-fibres," The British Journal of Surgery, vol. 42, no. 175, pp. 474-488, 1955.

[17] L. Montserrat and M. Benito, "Facial synkinesis and aberrant regeneration of facial nerve," Advances in Neurology, vol. 49, pp. 211-224, 1988.

[18] A. J. Sumner, "Aberrant reinnervation," Muscle and Nerve, vol. 13, no. 9, pp. 801-803, 1990 .

[19] M. Ito and M. Kudo, "Reinnervation by axon collaterals from single facial motoneurons to multiple muscle targets following axotomy in the adult guinea pig," Acta Anatomica, vol. 151, no. 2, pp. 124-130, 1994.

[20] C. L. A. M. Vleggeert-Lankamp, G. C. W. de Ruiter, J. F. C. Wolfs et al., "Type grouping in skeletal muscles after experimental reinnervation: Another explanation," European Journal of Neuroscience, vol. 21, no. 5, pp. 1249-1256, 2005.

[21] M. Streppel, N. Azzolin, S. Dohm et al., "Focal application of neutralizing antibodies to soluble neurotrophic factors reduces collateral axonal branching after peripheral nerve lesion," European Journal of Neuroscience, vol. 15, no. 8, pp. 1327-1342, 2002.

[22] M. Grosheva, O. Guntinas-Lichius, S. K. Angelova et al., "Local stabilization of microtubule assembly improves recovery of facial nerve function after repair," Experimental Neurology, vol. 209, no. 1, pp. 131-144, 2008.

[23] O. Guntinas-Lichius, A. Irintchev, M. Streppel et al., "Factors limiting motor recovery after facial nerve transection in the rat: combined structural and functional analyses," European Journal of Neuroscience, vol. 21, no. 2, pp. 391-402, 2005.

[24] P. Tos, B. Battiston, S. Geuna et al., "Tissue specificity in rat peripheral nerve regeneration through combined skeletal muscle and vein conduit grafts," Microsurgery, vol. 20, no. 2, pp. 65-71, 2000.

[25] U. Ozsoy, B. M. Demirel, A. Hizay et al., "Hypoglossalfacial anastomosis (HFA) over a $10 \mathrm{~mm}$ gap bridged by a Ytube-conduit enhances neurite regrowth and reduces collateral axonal branching at the lesion site," Restorative Neurology and Neuroscience, vol. 29, no. 4, pp. 227-242, 2011.

[26] E. Skouras, U. Ozsoy, L. Sarikcioglu, and D. N. Angelov, "Intrinsic and therapeutic factors determining the recovery of motor function after peripheral nerve transection," Annals of Anatomy, vol. 193, no. 4, pp. 286-303, 2011.

[27] A. Hizay, U. Ozsoy, B. M. Demirel et al., "Use of a Y-tube conduit after facial nerve injury reduces collateral axonal branching at the lesion site but neither reduces polyinnervation of motor endplates nor improves functional recovery," Neurosurgery, vol. 70, no. 6, pp. 1544-1556, 2012.

[28] K. Semba, H. Szechtman, and B. R. Komisaruk, "Synchrony among rhythmical facial tremor, neocortical "alpha" waves, and thalamic non-sensory neuronal bursts in intact awake rats," Brain Research, vol. 195, no. 2, pp. 281-298, 1980.
[29] R. W. Berg and D. Kleinfeld, "Rhythmic whisking by rat: retraction as well as protraction of the vibrissae is under active muscular control," Journal of Neurophysiology, vol. 89, no. 1, pp. 104-117, 2003.

[30] K. Semba and B. R. Komisaruk, "Neural substrates of two different rhythmical vibrissal movements in the rat," Neuroscience, vol. 12, no. 3, pp. 761-774, 1984.

[31] J. Dorfl, "The innervation of the mystacial region of the white mouse. A topographical study," Journal of Anatomy, vol. 142, pp. 173-184, 1985.

[32] G. E. Carvell and D. J. Simons, "Biometric analyses of vibrissal tactile discrimination in the rat," Journal of Neuroscience, vol. 10, no. 8, pp. 2638-2648, 1990.

[33] D. N. Angelov, O. Guntinas-Lichius, K. Wewetzer, W. F. Neiss, and M. Streppel, "Axonal branching and recovery of coordinated muscle activity after transection of the facial nerve in adult rats," in Advances in Anatomy Embryology and Cell Biology, F. F. Beck, B. Christ, F. Clascá et al., Eds., pp. 3-25, Springer, Berlin, Germany, 2005.

[34] A. Gruart, P. Blazquez, and J. M. Delgado-Garcia, "Kinematics of spontaneous, reflex, and conditioned eyelid movements in the alert cat," Journal of Neurophysiology, vol. 74, no. 1, pp. 226248, 1995.

[35] A. Gruart, M. Streppel, O. Guntinas-Lichius, D. N. Angelov, W. F. Neiss, and J. M. Delgado-García, "Motoneuron adaptability to new motor tasks following two types of facial-facial anastomosis in cats," Brain, vol. 126, no. 1, pp. 115-133, 2003.

[36] S. K. Koekkoek, W. L. den Ouden, G. Perry, S. M. Highstein, and C. I. de Zeeuw, "Monitoring kinetic and frequency-domain properties of eyelid responses in mice with magnetic distance measurement technique," Journal of Neurophysiology, vol. 88, no. 4, pp. 2124-2133, 2002.

[37] B. W. Quist and M. J. Z. Hartmann, "A two-dimensional force sensor in the millinewton range for measuring vibrissal contacts," Journal of Neuroscience Methods, vol. 172, no. 2, pp. 158-167, 2008.

[38] R. B. Towal and M. J. Z. Hartmann, "Variability in velocity profiles during free-air whisking behavior of unrestrained rats," Journal of Neurophysiology, vol. 100, no. 2, pp. 740-752, 2008.

[39] H. Bendella, S. P. Pavlov, M. Grosheva et al., "Non-invasive stimulation of the vibrissal pad improves recovery of whisking function after simultaneous lesion of the facial and infraorbital nerves in rats," Experimental Brain Research, vol. 212, no. 1, pp. 65-79, 2011.

[40] R. Bermejo, M. Harvey, P. Gao, and H. P. Zeigler, "Conditioned whisking in the rat," Somatosensory and Motor Research, vol. 13, no. 3-4, pp. 225-233, 1996.

[41] R. Bermejo, D. Houben, and H. P. Zeigler, "Optoelectronic monitoring of individual whisker movements in rats," Journal of Neuroscience Methods, vol. 83, no. 2, pp. 89-96, 1998.

[42] R. Bermejo and H. P. Zeigler, "Real-time' monitoring of vibrissa contacts during rodent whisking," Somatosensory and Motor Research, vol. 17, no. 4, pp. 373-377, 2000.

[43] T. Hadlock, J. Kowaleski, S. Mackinnon, and J. T. Heaton, "A novel method of head fixation for the study of rodent facial function," Experimental Neurology, vol. 205, no. 1, pp. 279-282, 2007.

[44] R. Leal-Campanario, J. A. Barradas-Bribiescas, J. M. DelgadoGarcía, and A. Gruart, "Relative contributions of eyelid and eyeretraction motor systems to reflex and classically conditioned blink responses in the rabbit," Journal of Applied Physiology, vol. 96, no. 4, pp. 1541-1554, 2004. 
[45] J. M. Delgado-Garcia, C. Evinger, M. Escudero, and R. Baker, "Behavior of accessory abducens and abducens motoneurons during eye retraction and rotation in the alert cat," Journal of Neurophysiology, vol. 64, no. 2, pp. 413-422, 1990.

[46] W. F. Crandall, S. J. Goldberg, J. S. Wilson, and J. R. McClung, "Muscle units divided among retractor bulbi muscle slips and between the lateral rectus and retractor bulbi muscles in cat," Experimental Neurology, vol. 71, no. 2, pp. 251-260, 1981.

[47] Y. Oda, "Extraocular muscles and their relationship to the accessory abducens nucleus in rats as studied by the horseradish peroxidase method," Okajimas Folia Anatomica Japonica, vol. 58, no. 1, pp. 43-53, 1981.

[48] S. Khanna and J. D. Porter, "Evidence for rectus extraocular muscle pulleys in rodents," Investigative Ophthalmology and Visual Science, vol. 42, no. 9, pp. 1986-1992, 2001.

[49] J. Husseman and R. P. Mehta, "Management of synkinesis," Facial Plastic Surgery, vol. 24, no. 2, pp. 242-249, 2008.

[50] C. H. G. Beurskens and P. G. Heymans, "Mime therapy improves facial symmetry in people with long-term facial nerve paresis: a randomised controlled trial," Australian Journal of Physiotherapy, vol. 52, no. 3, pp. 177-183, 2006.

[51] R. W. Lindsay, M. Robinson, and T. A. Hadlock, "Comprehensive facial rehabilitation improves function in people with facial paralysis: a 5-year experience at the Massachusetts eye and ear infirmary," Physical Therapy, vol. 90, no. 3, pp. 391-397, 2010.

[52] G. Borodic, M. Bartley, W. Slattery et al., "Botulinum toxin for aberrant facial nerve regeneration: double-blind, placebocontrolled trial using subjective endpoints," Plastic and Reconstructive Surgery, vol. 116, no. 1, pp. 36-43, 2005.

[53] D. K. Henstrom, J. S. Malo, M. L. Cheney, and T. A. Hadlock, "Platysmectomy: an effective intervention for facial synkinesis and hypertonicity," Archives of Facial Plastic Surgery, vol. 13, no. 4, pp. 239-243, 2011.

[54] M. H. Hohman, L. N. Lee, and T. A. Hadlock, "Two-step highly selective neurectomy for refractory periocular synkinesis," Laryngoscope, vol. 123, no. 6, pp. 1385-1388, 2013.

[55] B. Hontanilla, C. Aubá, J. Arcocha, and O. Gorría, "Nerve regeneration through nerve autografts and cold preserved allografts using tacrolimus (FK506) in a facial paralysis model: a topographical and neurophysiological study in monkeys," Neurosurgery, vol. 58, no. 4, pp. 768-777, 2006.

[56] R. N. Toledo, A. Borin, O. L. M. Cruz, P. L. Ho, J. R. G. Testa, and Y. Fukuda, "The action of topical basic fibroblast growth factor in facial nerve regeneration," Otology and Neurotology, vol. 31, no. 3, pp. 498-505, 2010.

[57] M. Wiberg, S. Vedung, and E. Stålberg, "Neuronal loss after transsection of the facial nerve: a morphological and neurophysiological study in monkeys," Scandinavian Journal of Plastic and Reconstructive Surgery and Hand Surgery, vol. 35, no. 2, pp. 135-140, 2001.

[58] A. Irintchev, "Potentials and limitations of peripheral nerve injury models in rodents with particular reference to the femoral nerve," Annals of Anatomy, vol. 193, no. 4, pp. 276-285, 2011.

[59] G. Ronchi, S. Nicolino, S. Raimondo et al., "Functional and morphological assessment of a standardized crush injury of the rat median nerve," Journal of Neuroscience Methods, vol. 179, no. 1, pp. 51-57, 2009.

[60] G. K. Frykman, P. J. McMillan, and S. Yegge, "A review of experimental methods measuring peripheral nerve regeneration in animals," Orthopedic Clinics of North America, vol. 19, no. 1, pp. 209-219, 1988.
[61] J. W. Griffin, B. Pan, M. A. Polley, P. N. Hoffman, and M. H. Farah, "Measuring nerve regeneration in the mouse," Experimental Neurology, vol. 223, no. 1, pp. 60-71, 2010.

[62] M. Brecht, V. Grinevich, T.-E. Jin, T. Margrie, and P. Osten, "Cellular mechanisms of motor control in the vibrissal system," Pflugers Archiv European Journal of Physiology, vol. 453, no. 3, pp. 269-281, 2006.

[63] M. Brecht, B. Preilowski, and M. M. Merzenich, "Functional architecture of the mystacial vibrissae," Behavioural Brain Research, vol. 84, no. 1-2, pp. 81-97, 1997.

[64] R. N. S. Sachdev, T. Sato, and F. F. Ebner, "Divergent movement of adjacent whiskers," Journal of Neurophysiology, vol. 87, no. 3, pp. 1440-1448, 2002.

[65] T. Berg, L. Jonsson, and M. Engström, "Agreement between the Sunnybrook, House-Brackmann, and Yanagihara facial nerve grading systems in Bell's palsy," Otology and Neurotology, vol. 25, no. 6, pp. 1020-1026, 2004.

[66] C. H. Yian, R. C. Paniello, and J. Gershon Spector, "Inhibition of motor nerve regeneration in a rabbit facial nerve model," Laryngoscope, vol. 111, no. 5, pp. 786-791, 2001.

[67] J. M. Byers, K. F. Clark, and G. C. Thompson, "Effect of pulsed electromagnetic stimulation on facial nerve regeneration," Archives of Otolaryngology: Head and Neck Surgery, vol. 124, no. 4, pp. 383-389, 1998.

[68] S. D. de Faria, J. R. G. Testa, A. Borin, and R. N. Toledo, "Standardization of techniques used in facial nerve section and facial movement evaluation in rats," Revista Brasileira de Otorrinolaringologia, vol. 72, no. 3, pp. 341-347, 2006.

[69] T. Hadlock, J. Kowaleski, D. Lo et al., "Functional assessments of the rodent facial nerve: a synkinesis model," Laryngoscope, vol. 118, no. 10, pp. 1744-1749, 2008. 

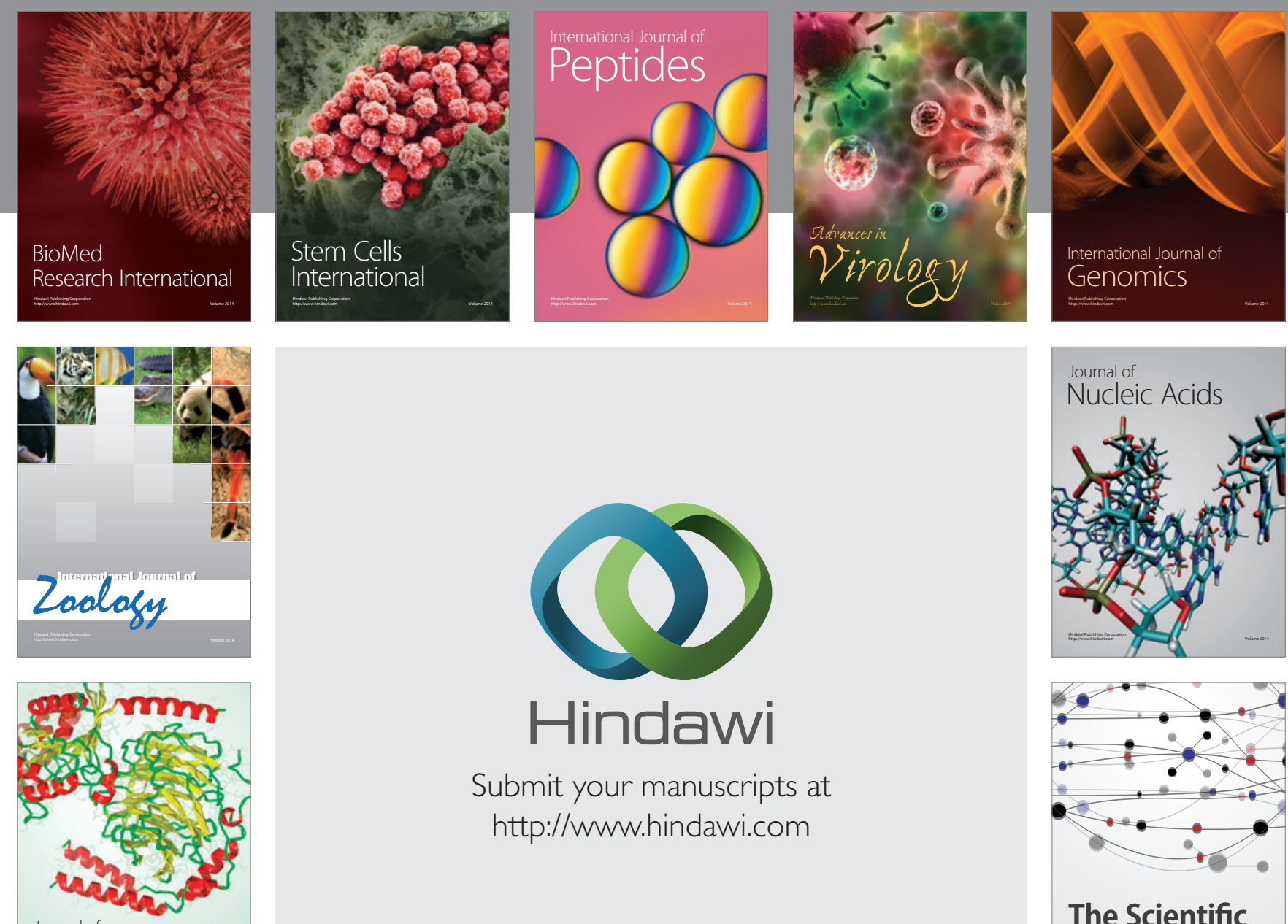

Submit your manuscripts at

http://www.hindawi.com

Journal of
Signal Transduction
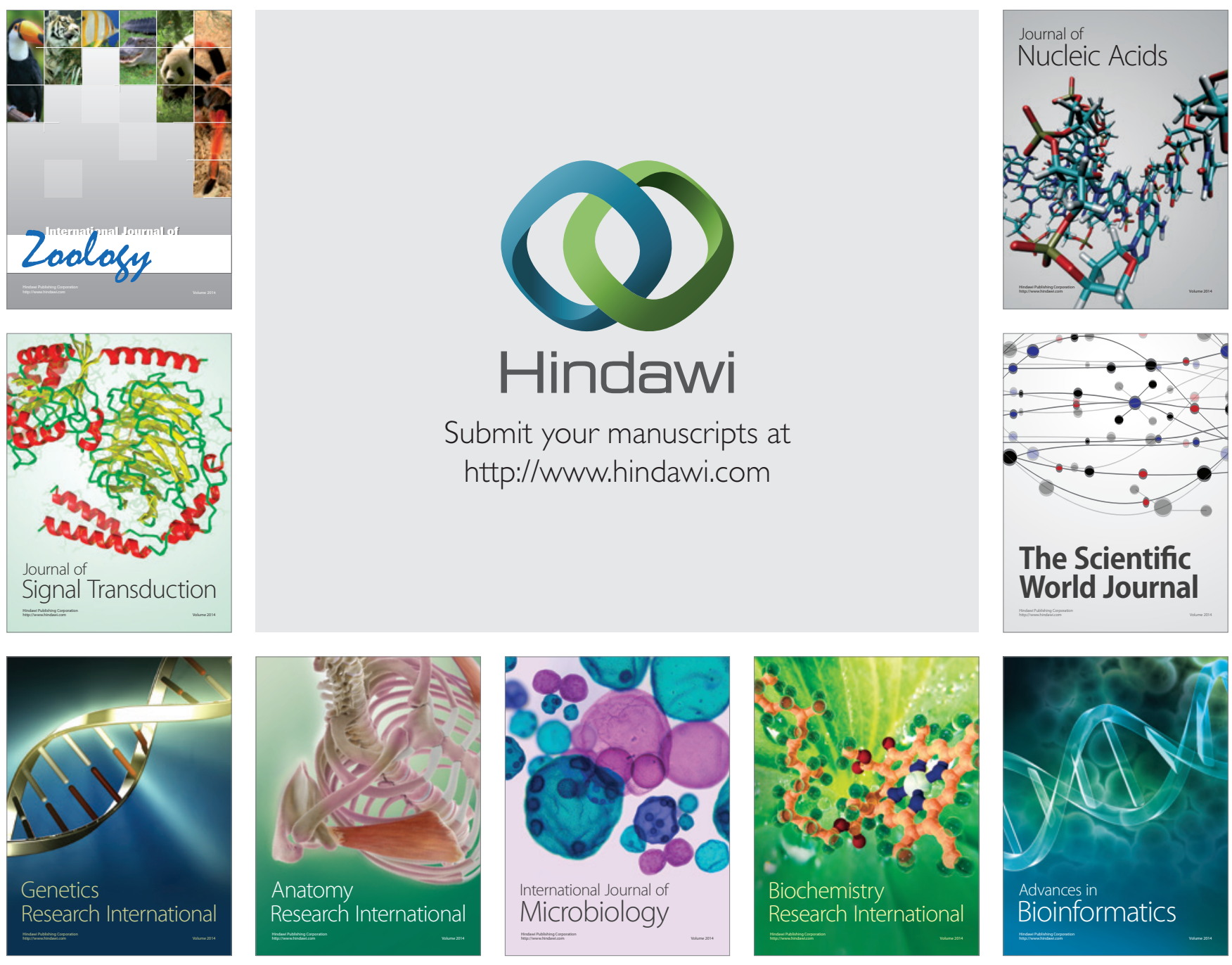

The Scientific World Journal
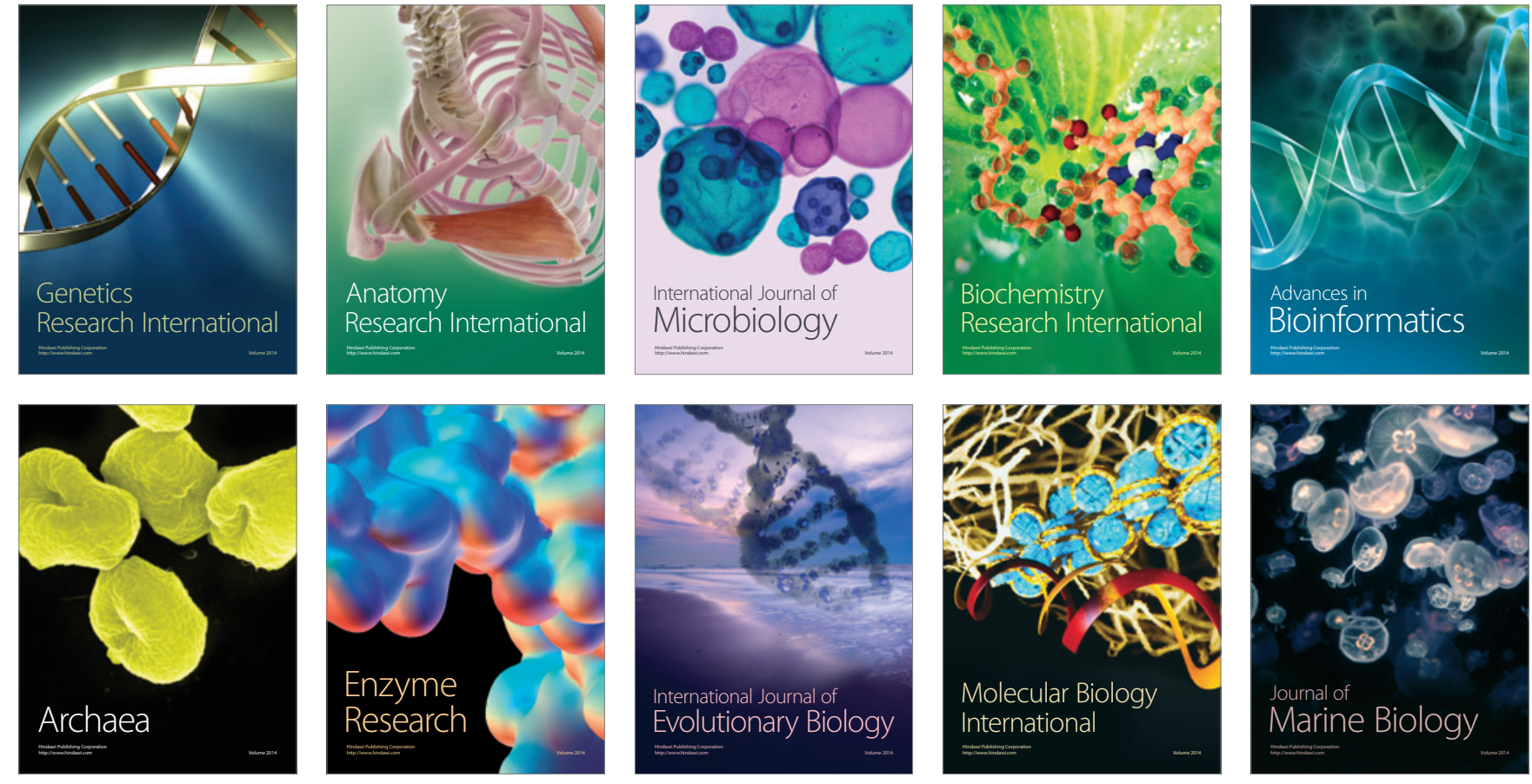